\title{
FITOTERAPIA, ALLIUM SATIVUM E HIPERCOLESTEROLEMIA: UMA REVISÃO
}

\author{
PHYTOTHERAPY, ALLIUM SATIVUM AND HYPERCHOLESTEROLEMIA: A REVIEW
}

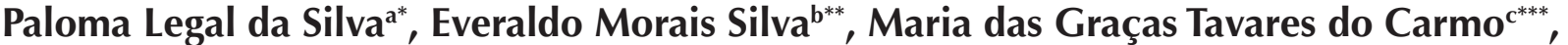 Felipe de Souza Cardoso ${ }^{\mathrm{d}^{* *}}$}

\author{
apalomalegall@yahoo.com.br, beveraldofarma@yahoo.com.br, ctcarmo@editema.com.br, dfelipe.souza.cardoso@hotmail.com \\ *Instituto de Pesquisas Ensino e Gestão em Saúde - Porto Alegre (RS), Brasil \\ **Faculdade Bezerra de Araújo - Rio de Janeiro (RJ), Brasil \\ ***Universidade Federal do Rio de Janeiro - Rio de Janeiro (RJ), Brasil
}

Data de recebimento: 05/12/2015 Data de aceite: 04/03/2016

\section{RESUMO}

Introduçáo: $\mathrm{Na}$ atualidade, as doenças crônicas não transmissíveis são consideradas grande problema de saúde pública. No Brasil, a principal causa de morte é por doença arterial coronariana. Entre os fatores de risco, o colesterol vem sendo apontado tanto em evidências observacionais quanto em experimentais pré-clínicas e clínicas como o principal fator de risco modificável. O objetivo desse estudo de revisão foi avaliar a ação da espécie Allium sativum sobre o perfil lipídico sanguíneo de adultos com dislipidemia. Desenvolvimento: Foi realizada uma revisão da literatura nos seguintes bancos de dados: SciELO e PubMed. O idioma de pesquisa foi o inglês através dos seguintes descritores: "Allium sativum", "hypercholesterolaemia", "dyslipidemia", "cardiovascular diseases" e "chronic diseases". O estudo priorizou artigos entre os anos de 1993 e 2014, não excluindo artigos considerados clássicos. $\mathrm{O}$ número amostral das pesquisas avaliadas com Allium sativum variou entre 15 e 192, tendo sido empregadas, nas intervençóes, doses desde a forma crua até extrato padronizado, que variaram entre 5 e $7200 \mathrm{mg}$ de alho total. Apesar das limitaçóes metodológicas observadas, como oscilações entre as doses, formas de apresentação e amostra reduzida, 58\%, aproximadamente, indicaram benefícios da utilização do alho sobre o controle do perfil lipídico, principalmente no que diz respeito à alteração de colesterol total e lipoproteína de baixa densidade, sugerindo que a substância alicina, presente em relação à quantidade do alho cru, pó ou extrato total, seria a principal moduladora dos efeitos observados sobre o perfil lipídico. Observou-se que a reduçáo de colesterol total variou entre 4,2 e 12,1\%, e a variação na redução de lipoproteína de baixa densidade entre 6,6 e 17,6\%. Conclusão: Allium sativum parece ser uma alternativa eficaz e segura sobre a redução da hipercolesterolemia. Entretanto, pesquisas que demonstrem evidências clínicas são necessárias para excluir as possíveis variáveis de confundimento.

Palavras-chave: Allium sativum; hipercolesterolemia; dislipidemia; doenças cardiovasculares; doença crônica.

\section{ABSTRACT}

Introduction: Currently, chronic diseases are considered major public health problem. In Brazil, the main cause of death is due to coronary artery disease. Among the risk factors, cholesterol has been pointed out, both observational and experimental preclinical and clinical evidence, as the main modifiable risk factor. The objective of this review study was to evaluate the action of Allium sativum species on the lipid profile of adults with dyslipidemia. Development: conducted a literature review in the following databases: SciELO and PubMed. English language following keywords: "Allium sativum", "hypercholesterolaemia”, "dyslipidemia", "cardiovascular diseases" and "chronic diseases". The study prioritized items between the years 1993 and 2014, not excluding items considered classics. The sample size of the research evaluated Allium sativum ranged from 15 to 192, indicating from raw form to standardized extract, and dose ranging between 5 and $7200 \mathrm{mg}$ total garlic. Despite methodological limitations observed as oscillations between doses, presentations and small sample, 58\% approximately indicated benefits of using garlic over control of the lipid profile, particularly with regard to total cholesterol changes and lipoprotein low density, suggesting that the allicin substance present in the quantity of raw garlic powder or total extract would be the main modulator of the observed effects on the lipid profile. It was observed that the reduction of total cholesterol varies between 4.2 and $12.1 \%$, and the variation in reducing low density lipoprotein between 6.6 and $17.6 \%$. Conclusion: Allium sativum appears to be a safe and effective alternative for reducing hypercholesterolemia. However, research demonstrating clinical evidence is necessary to exclude possible confounding variables.

Keywords: Allium sativum; hypercholesterolaemia; dyslipidemia; cardiovascular diseases; chronic diseases. 


\section{Introdução}

Nas últimas décadas, as doenças crônicas não transmissíveis (DCNT) tornaram-se grande problema de saúde pública ${ }^{1}$. Em 2007, segundo dados da Organização Mundial de Saúde (OMS), DCNT foram responsáveis por $59 \%$ do total de mortes no mundo e há uma previsão de que ultrapassem em $78 \%$ dessas mortes em $2020^{2}$. Esses dados, ainda, são mais alarmantes nos países de baixa e média renda, onde cerca de $80 \%$ das mortes já são derivadas das DCNT e 29\% ocorrem em indivíduos abaixo de 60 anos. Entretanto, em países desenvolvidos, essa taxa de morte prematura cai para $13 \%{ }^{2}$. Entre as DCNT, a principal causa de morte no Brasil é por Doença Arterial Coronariana (DAC), em grande parte associada a alterações dietéticas, nas fraçōes de colesterol sanguíneo, apontadas em evidências observacionais e experimentais ${ }^{3}$. De acordo com dados epidemiológicos, uma redução em $10 \%$ da fração de lipoproteína de baixa densidade (LDL) resultaria em benefício de $20 \%$ sobre o risco de desenvolvimento para doença cardíaca ao longo da vida ${ }^{4}$.

Os tratamentos disponíveis para dislipidemia são diversos, desde as condutas clássicas com medicação alopática sintética, dieta, correçóes no estilo de vida (perda de peso, atividade física regular, cessaçáa do tabagismo etc.) até as condutas integrativas ${ }^{3}$. Os principais tratamentos medicamentosos são feitos com estatinas, resinas e ezetimiba, mas as estatinas continuam em primeira linha, agindo sobre a inibição da enzima Hidroximetilglutaril Coenzima A redutase (HMG CoA redutase), e associadas a possíveis efeitos adversos, como miopatia (após anos de utilização), toxicidade hepática etc. A resina age como redutor de ácidos biliares, com efeitos colaterais como constipaçâo intestinal e aumento dos triglicerídeos. A ezetimiba inibe a absorção de colesterol na borda em escova do intestino delgado, sendo correlacionada, então, à alteração do trânsito intestinal e à esteatorreia ${ }^{3}$. A fitoterapia, assim, apresenta-se como mais uma alternativa na prevenção e tratamento da dislipidemia ${ }^{3}$.

A utilização da fitoterapia como complementar à dietoterapia é regulamentada pela resolução no 525/13 do Conselho Federal de Nutricionistas ${ }^{5}$, apoiada em resoluçóes da Agência Nacional de Vigilância Sanitária (ANVISA) como a RDC n ${ }^{\circ} 10 / 10^{6}$ e IN no $02 / 14^{7}$. Todos os documentos regulatórios apoiam a utilização racional, baseada nos critérios de eficácia e segurança. Portanto, fornecem informaçóes fundamentadas em evidências e tradicionalismo comprovado, sem histórico de toxicidade importante, e contemplando seus possíveis efeitos adversos.

Entre as diversas espécies utilizadas nessa ciência, a Allium sativum está entre as mais antigas plantas cultivadas e utilizadas com diversos fins: especiarias na culinária, fitoterapia, espiritualidade etc. Neste texto, a espécie Allium sativum é indicada por suas possíveis propriedades terapêuticas. $\mathrm{O}$ alho teve a sua origem nas estepes da Ásia Central, de onde se expandiu para o leste, até a China, e, depois, para o oeste, chegando à Europa ${ }^{8}$. As primeiras apariçóes foram encontradas em tabuletas de argila suméricas, datadas em 2600 a 2100 anos a.C. ${ }^{8,9}$. O alho foi amplamente utilizado como medicamento, listados em textos médicos Codex Ebers de aproximadamente 1550 anos a.C, pelos antigos povos egípcios. Uma das suas indicaçóes, descritas neste texto, está correlacionada ao tratamento das doenças do sistema circulatório, mal-estar em geral, picadas de insetos e infestaçóes por parasitas, tendo sido, também, utilizado pela classe trabalhadora envolvida com a construção das pirâmides a fim de aumentar sua força e produtivida$\mathrm{de}^{8}$. Evidências indicam que, durante os primeiros jogos olímpicos na Grécia, o alho foi utilizado por atletas com objetivo de aumentar a resistência ${ }^{9}$. Na medicina chinesa antiga, o alho foi utilizado como auxílio na respiração e digestão, e como combate a diarreias e a infestações por vermes ${ }^{10}$. $\mathrm{Na}$ Índia, tradiçóes médicas antigas, ou seja, Tibb, Unani e Ayurveda, fizeram uso extensivo de alho como parte central da eficácia da cura através das plantas $^{8}$. Na Renascença, a Europa voltou sua atenção para a prescrição médica de plantas medicinais, e o alho foi uma das principais plantas cultivadas. Um médico líder do século XVI, Pietro Mattioli, de Siena, prescrevia alho para distúrbios digestórios, infestação de vermes e doenças renais, bem como para ajudar mães durante partos mais complicados. Na América, o alho foi trazido da França e Portugal pelos colonizadores. A partir do século XIX, o alho ganhou destaque ao começar a fazer parte do arsenal médico de Shaker como estimulante, expectorante e tônico, tendo, assim, reconhecidas suas propriedades terapêuticas por grande parte da população ${ }^{8}$.

A composição química do alho indica presença de aliina/alicina (após conversão pela aliinase) ${ }^{9}$, água, proteínas, lipídeos, carboidratos, fibra alimentar, cálcio, ferro, magnésio, fósforo, potássio, sódio, zinco, cobre, manganês, selênio, vitamina $\mathrm{C}$, tiamina, riboflavina, niacina, ácido pantotênico, vitamina B6, colina, luteína, zeaxantina, vitamina $\mathrm{E}$ e vitamina $\mathrm{K}^{11}$. Dentro desse contexto, justifica-se a ampla utilizaçáo da espécie Allium sativum como moduladora de uma variabilidade de efeitos biológicos ${ }^{12,13}$.

A alicina, precursora de alguns produtos como ajoeno, vinilditiinas, oigosulfidos e polisulfidos, saponinas, proteínas, sais minerais, ácidos fosfóricos e sulfúricos ${ }^{12,13}$, indica, em evidências, atividade antitrombótica, antiplaquetária ${ }^{12,14}$, antioxidante ${ }^{15}$, antibactericida $^{16}$, hipocolesterolêmicas ${ }^{17}$, hipoglicemiante ${ }^{12,14,18}$ 
e hipotensora ${ }^{12,17,19}$. A maioria das pesquisas, tanto observacionais quanto experimentais, tem reforçado os efeitos positivos sobre os fatores de risco cardiovascular, pois reduz a hiperlipidemia e a hipertensão, além de prevenir a formação de trombos. Pesquisadores, então, têm apoiado o uso de alho na prevenção de doenças cardiovasculares, motivados, também, pelos poucos efeitos secundários, como mau hálito e problemas digestórios ${ }^{20}$. Em função disso, este trabalho de revisão da literatura propóe verificar a ação da espécie Allium sativum sobre a redução da hipercolesterolemia em adultos, já que esse é o principal fator de risco modificável associado à doença arterial coronariana, principal causa de morte dentro das $\mathrm{DCNT}^{3}$.

\section{Desenvolvimento}

\section{Evidências sobre os efeitos da espécie Allium sativum no perfil lipídico}

A pesquisa foi desenvolvida com base nos seguintes bancos de dados: PubMed e SciELO. O idioma de pesquisa foi o inglês através dos seguintes descritores: "Allium sativum", "hypercholesterolaemia", "dyslipidemia", "cardiovascular diseases" e "chronic diseases". Priorizaram-se artigos entre os anos de 1993 e 2014 em função do número restrito de publicações clínicas sobre o assunto. Foram considerados somente artigos com delineamento experimental clínico em adultos, com o alho, nas suas diferentes formas de apresentação farmacêutica, como intervenção sobre o perfil lipídico e/ou sobre desfechos correlatos, totalizando 14 publicaçóes analisadas. Todas as evidências foram interpretadas de acordo com a ordem cronológica de publicação para facilitar o entendimento da evoluçáo.

No estudo de Jain et al. ${ }^{21}, 42$ adultos saudáveis (19 homens e 23 mulheres), com idade média de $52( \pm 12)$ anos, valor de colesterol total (CT) igual ou acima de $220 \mathrm{mg} / \mathrm{dL}$, em estudo randomizado e duplo-cego, receberam $300 \mathrm{mg}$ de alho em pó três vezes ao dia, em comprimidos, com dietas e atividade física inalteradas. As diferenças entre o CT da linha de base e após 12 semanas de tratamento foi de $15 \mathrm{mg} / \mathrm{dL}(\mathrm{p}<0,01)$. Os valores correspondentes ao placebo foram $276( \pm 34$; antes) e 274 ( \pm 29 ; após). A LDL foi reduzida em $11 \%$ para o tratamento e em $3 \%$ para o grupo placebo $(p<0,05)$ entre o início e o final do estudo ${ }^{21}$.

Steiner et al. ${ }^{22}$, em estudo cruzado e duplo-cego, compararam o efeito do extrato de alho envelhecido com placebo sobre o perfil lipídico. Para isso, trabalharam com um grupo de 41 homens moderadamente hipercolesterolêmicos $(220-290 \mathrm{mg} / \mathrm{dL})$. Após um período de quatro semanas, no qual os participantes foram conduzidos sobre a importância da adesáo à dieta nacional do programa de educação em colesterol, iniciou-se o tratamento com 7,2 g por dia de extrato de alho envelhecido ou uma quantidade equivalente de placebo, durante seis meses. As principais alteraçôes foram em relação à redução de 7\% de CT, em comparação à linha de base. A LDL também indicou redução, assim como $5,5 \%$ da pressão arterial sistólica ${ }^{22}$.

Adler et al. ${ }^{23}$ examinaram os efeitos do alho, em jejum, sobre o perfil lipídico plasmático em indivíduos hipercolesterolêmicos. Aleatoriamente, 50 indivíduos do sexo masculino com hipercolesterolemia moderada foram distribuídos em quatro grupos. Um dos grupos avaliou o efeito do alho isoladamente, administrando $900 \mathrm{mg}$ de alho em pó. A concentração média de CT do grupo tratado foi significativamente menor com o alho $(-11,5 \%)$, após 12 semanas. A média da concentração de LDL foi reduzida $(-14,2 \%)$. Portanto, nesse estudo, o alho diminuiu significativamente tanto o CT quanto a $\mathrm{LDL}^{23}$.

Garden et al. ${ }^{24}$ trabalharam com 51 adultos, idade de 51,8 anos $( \pm 8,3)$, em ensaio clínico randomizado, placebo controlado e duplo-cego. Foram fornecidos 500 ou $1000 \mathrm{mg}$ de alho em pó desidratado por dia. O perfil lipídico avaliado após 12 semanas indicou redução de LDL em 6,1\%; entretanto, não significativamente, diferente dos outros grupos $(\mathrm{p}=0,5)$. Não foram observadas diferenças significativas para CT, HDL e TG ${ }^{24}$.

Kannar et al. ${ }^{25}$, em seu estudo de intervençáo controlado com placebo, duplo-cego e randomizado, contemplaram 46 pacientes com hipercolesterolemia, que não estavam em conformidade com a terapia medicamentosa. Cada sujeito recebeu aconselhamento dietético para reduzir a ingestão de gorduras, além de comprimidos de alho em pó $(9,6 \mathrm{mg})$ ou placebo. Após 12 semanas, o grupo tratado $(\mathrm{n}=22)$ indicou redução significativa sobre o CT $(-4,2 \%)$ e LDL (-6.6\%), enquanto o grupo placebo $(\mathrm{n}=24)$ mostrou aumento não significativo. A HDL foi significativamente aumentada no grupo placebo $(9,1 \%)$. Demonstrou-se, então, que o alho em pó pode ter relevância clínica em pacientes com hipercolesterolemia, quando combinado à dieta com teor de gordura reduzido. Entretanto, a eficácia no metabolismo das lipoproteínas poderia "exigir" um trabalho para a biodisponibilidade de alicina, como foi o caso dos comprimidos. Também digna de nota foi a pequena redução do consumo de energia no grupo que consumiu o alho em comparação ao placebo, atribuível, provavelmente, à redução de gordura, carboidratos e ingestão de álcool, o que pode ter contribuído para os efeitos sobre os lipídios no sangue ${ }^{25}$.

Peleg et al. ${ }^{26}$ realizaram um estudo, durante 16 semanas, controlado por placebo, duplo-cego, envolvendo 33 pacientes com hipercolesterolemia primária e sem evidência de doença cardiovascular, os quais foram 
designados, aleatoriamente, a receber alho $(22,4 \mathrm{mg}$ aliina) ou placebo. Ambos os grupos receberam aconselhamento dietético individual, e as alteraçóes no perfil lipídico foram determinadas no início e no final do estudo. Não foram observadas alteraçôes significativas nos níveis de CT, LDL, HDL e TG ${ }^{26}$.

Satitvipawee et al. ${ }^{27}$ realizaram um estudo duplo-cego, randomizado, controlado por placebo, com 136 pacientes diagnosticados com hipercolesterolemia (concentraçôes de colesterol $\geq 5,2 \mathrm{mmol} / \mathrm{L}$ ). Os pacientes apresentavam média de 47 anos de idade ( $\pm 6,6$ anos). Todos os participantes receberam, durante quatro semanas, aconselhamento dietético para a ingestão controlada de gorduras e foram aconselhados a manter a alimentaçáo normal durante o período do estudo. Os indivíduos foram, aleatoriamente, designados para receber uma vez por dia, após a refeição da noite, durante 12 semanas, um extrato de alho em comprimido de 5,6 $\mathrm{mg}$ (padronizado para $1,12 \%$ de alicina, assim como o placebo). Um total de 70 indivíduos $(32,9 \%$ do sexo masculino; IMC $\left.=24,6 \pm 3,3 \mathrm{~kg} / \mathrm{m}^{2}\right)$ recebeu o tratamento, enquanto 66 indivíduos (37,9\% do sexo masculino; IMC $=24,3 \pm 3,4 \mathrm{~kg} / \mathrm{m}^{2}$ ) receberam placebo. Não houve alteraçóes estatisticamente significativas para os níveis de CT, TG, LDL e HDL após as 12 semanas $^{27}$.

Tanamai et al. ${ }^{28}$ conduziram 100 voluntários em estudo aleatório, cruzado e duplo-cego, os quais foram divididos em dois grupos: 45 no grupo tratado e 55 no controle. Os voluntários do grupo tratado receberam comprimidos de alho nos primeiros três meses, placebo nos próximos três meses e interromperam todos os comprimidos nos últimos três meses, totalizando nove meses. O grupo controle começou com três meses de placebo, seguindo em três meses de comprimidos de alho e terminando em três meses de descontinuidade. Os resultados indicaram que não existiam diferenças significativas nos níveis de CT entre os dois grupos ao fim do estudo ${ }^{28}$.

Participaram do estudo de Sumioka et al. ${ }^{29} 15$ pessoas, com idades entre 33-59 anos (11 homens e 4 mulheres). Todos tinham CT ( $\geq 220 \mathrm{mg} / \mathrm{dL})$ ou VLDL ( $\geq 140$ $\mathrm{mg} / \mathrm{dL})$, mas apenas 4 dos $15(27 \%)$ apresentavam hipertrigliceridemia $(\geq 150 \mathrm{mg} / \mathrm{dL})$. Os participantes receberam duas cápsulas de $225 \mathrm{mg} /$ dia após o almoço e o jantar, durante 4 semanas. O sangue de cada um foi avaliado durante o tratamento e duas semanas após a retirada da intervenção. O CT indicou redução estatisticamente significativa, assim como a LDL e o índice aterogênico, calculado a partir do valor de TC e o HDL-C após quatro semanas, comparado à linha de base. Esses efeitos tenderam ao desaparecimento após duas semanas de retirada das cápsulas ${ }^{29}$.

Macan et al. ${ }^{30}$ trabalharam com 66 pacientes em estudo piloto, controlado por placebo, duplo-cego e randomizado. Desses, 52 foram, aleatoriamente, selecionados para o projeto. 48 pacientes (30 homens e 18 mulheres), com idade média de 56 ( \pm 10 anos) completaram o estudo. Dezoito pacientes (14 através da randomização e 4 após a randomizaçáo) foram retirados do estudo. $\mathrm{O}$ tratamento ou placebo foram administrados em doses de $5 \mathrm{~mL}$, duas vezes por dia, durante 12 semanas. Sangramento potencial e episódios tromboembólicos foram monitorados. Não havia nenhuma evidência de aumento da hemorragia em ambos os grupos. Os resultados sugeriram que a idade é relativamente segura e não indica risco hemorrágico grave para pacientes monitorados, em terapia de anticoagulação oral, com varfarina ${ }^{30}$.

Gardner et al. ${ }^{31}$, em seu ensaio clínico envolvendo 192 adultos em concentraçóes entre 130 a 190 mg de LDL, distribuíram, aleatoriamente, todos os participantes em quatro grupos de tratamento: alho cru, alho em pó, extrato envelhecido ou placebo. Não houve efeito estatisticamente significativo para as três formas de alho sobre as concentraçóes de LDL, HDL, TG e CT ${ }^{31}$.

No estudo controlado por placebo e duplo-cego de Kojuri et al. ${ }^{32}$, os perfis lipídicos de 150 pacientes hiperlipidêmicos foram verificados nas mesmas condiçóes. Os participantes foram divididos em três grupos iguais (cada um com 50 pacientes), de forma aleatória, todos em controle dietético. Receberam comprimidos de alho em pó ( $400 \mathrm{mg}$ de alho, $1 \mathrm{mg}$ alicina) duas vezes ao dia, em tabletes de $650 \mathrm{mg}$, assim como o placebo. Após o tratamento, o CT indicou redução de $12,1 \%(P=0,0008)$, e o LDL-colesterol de $17,3 \%(P=0,000)$, assim como ocorreu aumento de $\mathrm{HDL}$ de $15,7 \%(P=0,000)^{32}$.

Sobenin et al..$^{33}$ avaliaram os efeitos hipolipemiantes do alho em pó (600 mg por dia) em estudo randomizado, duplo-cego e controlado por placebo, envolvendo 42 homens com idades entre 35-70 e hipercolesterolêmicos. O tratamento resultou em diminuição moderada e estatisticamente significativa no nível de colesterol total após 12 semanas. Os níveis de CT dos tratados indicou redução de 7,6\% $(P=0,004)$ ao final do estudo, sendo $11,5 \%$ menor do que o grupo placebo $(P=0,005)$. A LDL reduziu $11,8 \%(P=0,002)$ e $13,8 \%(P=0,009)$, da mesma forma. O colesterol HDL também aumentou significativamente após o tratamento $(11,5 \% ; P=0,013)^{33}$.

Jung et al. ${ }^{34}$ realizaram estudo duplo-cego, controlado por placebo e randomizado, envolvendo 55 participantes (28 tratados e 27 placebos), designados para receber, aleatoriamente, o alho ou placebo, duas vezes ao dia (total $6 \mathrm{~g} / \mathrm{d}$ ), antes do consumo de uma refeição a cada manhã e à noite, durante 12 semanas. Sobre o perfil lipídico, não foram observadas diferenças significativas em TG, LDL, CT ou em níveis de ácidos graxos livres entre os dois grupos. No entanto, houve aumento dos níveis de HDL, em comparaçáo com o grupo placebo, no final do estudo, e diminuição significativa para os níveis de apolipoproteína $\mathrm{B}^{34}$. 
Na Tabela 1, são indicadas as reduçóes de CT e LDL, em percentual, com seus respectivos valores de significância estatística. Observa-se que a redução de CT varia entre 4,2 e $12,1 \%$, e, a de LDL, entre 6,6 e 17,6\%.

Tabela 1: Redução do colesterol total e da lipoproteína de baixa densidade após a intervenção.

\begin{tabular}{ccccc} 
Referência & \% CT & $P$ valor & \% LDL & p valor \\
$(23)$ & $-11,5$ & 0,05 & $-14,2$ & 0,05 \\
$(21)$ & $-5,7$ & 0,01 & -11 & 0,05 \\
$(33)$ & $-7,6$ & 0,004 & $-11,8$ & 0,002 \\
$(22)$ & $-7,0$ & 0,0001 & $\mathrm{x}$ & $\mathrm{x}$ \\
$(32)$ & $-12,1$ & 0,0001 & $-17,3$ & 0,0001 \\
$(29)$ & -20 & 0,01 & $-17,6$ & 0,01 \\
$(25)$ & $-4,2$ & $<0,05$ & $-6,6$ & $<0,05$ \\
\hline
\end{tabular}

CT: colesterol total; LDL: lipoproteína de baixa densidade; x: dados náo disponíveis.

A partir dos estudos avaliados, observa-se variedade de doses, formas de apresentação, reduzido número amostral e de efeitos. As amostras variaram entre $15 \mathrm{e}$ 192 , e receberam doses, empregadas desde a forma crua até a de extratos padronizados, que variaram entre $5 \mathrm{e}$ $7200 \mathrm{mg}$ de alho total, levantando à hipótese de que, entre elas, a alicina teria maior importância, se comparada à quantidade do alho cru, pó ou extrato total. No entanto, a maior parte indicou eficácia e segurança para duração de 12 semanas, em adultos (Tabela 2).

Tabela 2: Ensaios clínicos randomizados com humanos adultos.

\begin{tabular}{|c|c|c|c|c|c|c|}
\hline Ref. & $\mathbf{n}$ & Sexo & Forma & Dose (mg) & $\begin{array}{c}\text { Duraçáo } \\
\text { (sem) }\end{array}$ & Resultados \\
\hline (23) & 50 & $\mathrm{~h}$ & pó & $900^{*}$ & 12 & +CT, LDL \\
\hline (24) & 51 & $\mathrm{x}$ & pó & $\begin{array}{c}500- \\
1000^{*}\end{array}$ & 12 & - \\
\hline (21) & 42 & h e m & pó & 900 & 12 & +CT, LDL \\
\hline (26) & 33 & $\mathrm{he} \mathrm{m}$ & pó & $22,4^{* *}$ & 16 & - \\
\hline (33) & 42 & $\mathrm{~h}$ & pó & $600^{*}$ & 12 & +CT, LDL \\
\hline (22) & 41 & $\mathrm{~h}$ & extrato & $7200^{* * *}$ & 24 & $\begin{array}{l}\text { +CT, LDL, } \\
\text { PS, PD }\end{array}$ \\
\hline (32) & 150 & $\mathrm{x}$ & pó & 200 & 6 & $\begin{array}{c}+\mathrm{HDL},-\mathrm{CT}, \\
\mathrm{LDL}\end{array}$ \\
\hline (31) & 192 & $\mathrm{x}$ & $\begin{array}{c}\text { cru } \\
\text { pó } \\
\text { extrato }\end{array}$ & 4000 & 24 & $\begin{array}{c}\text {-HDL, -CT, } \\
\text { LDL }\end{array}$ \\
\hline (29) & 15 & he $\mathrm{m}$ & e ferm & 450 & 4 & $\begin{array}{c}\text {-CT, LDL, } \\
\text { IA }\end{array}$ \\
\hline (30) & 48 & h e m & extrato & 3050 & 12 & $\begin{array}{c}\text { - interação } \\
\text { varfarina }\end{array}$ \\
\hline (28) & 100 & h e $m$ & extrato & 5 & 12 & -CT, LDL \\
\hline (27) & 136 & $\mathrm{x}$ & extrato & 5,6 & 12 & -CT, LDL \\
\hline (25) & 46 & h e m & pó & 880 & 12 & +CT, LDL \\
\hline (34) & 55 & $\mathrm{x}$ & negro & 6000 & 12 & $\begin{array}{c}++\mathrm{HDL} \\
+ \text { +apoB }\end{array}$ \\
\hline
\end{tabular}

Ref.: referências; n: número de participantes.; h: homens; m: mulheres; mg: miligramas; CT: colesterol total; LDL: lipoproteína de baixa densidade; sem: semanas; PS: pressão sistólica; PD: pressão diastólica; +: redução; ++: aumento; -: nenhuma diferença; ${ }^{*}$ : mg de alho seco em pó; ${ }^{* *}$ : mg de aliina; ${ }^{* * *}$ : mg de extrato de alho; e ferm: extrato fermentado; IA: índice aterogênico; apoB: apolipoproteína B.

\section{Conclusão}

Existem poucas evidências sobre a utilização da espécie Allium sativum em casos de hipercolesterolemia, e os estudos são limitados, metodologicamente, em função da variedade dos parâmetros avaliados. Entretanto, em relação à toxicidade, a espécie parece ser eficaz e segura para a prática clínica, sendo, inclusive, recomendada pela ANVISA.

\section{Referências}

1. World Health Organization. Preventing chronic diseases: a vital investment. Geneva; 2005.

2. World Health Organization. Global status report on noncommunicable diseases 2010. Geneva; 2011.

3. Sociedade Brasileira De Cardiologia. V Diretriz Brasileira de Dislipidemias e Prevenção e da Aterosclerose. Arq Bras Cardiol. 2013;101(4):1-22.

4. Larosa JC. Low-density lipoprotein cholesterol reduction: the end is more important than the means. Am J Cardiol. 2007;100(2):240-42.

5. Brasil. Conselho Federal de Nutricionistas. Resoluçáo CFN n525, de 28 de junho de 2013. Regulamenta a prática da fitoterapia pelo nutricionista atribuindo-lhe competência para, nas modalidades que especifica, prescrever plantas medicinais, drogas vegetais e fitoterápicos como complemento da prescriçâo dietética e, dá outras providências. Brasília, DF; 2013.

6. Brasil. Agência Nacional de Vigilância Sanitária. Resolução RDC no10, de 9 de março de 2010. Dispóe sobre a notificação de drogas vegetais junto à Agencia Nacional de Vigilância Sanitária (Anvisa) e dá outras providências. Brasília, DF; 2010.

7. Brasil. Agência Nacional de Vigilância Sanitária. Instrução Normativa IN $n^{\circ} 02$, de 13 de maio de 2014. Publica a lista de medicamentos fitoterápicos de registro simplificado e a lista de produtos tradicionais fitoterápicos de registro simplificados. Brasília, DF; 2014.

8. Moyers S. Garlic in Health, History and World Cuisine. Suncoast Press, St. Petersburg; 1996. p. 1-36.

9. Lawson LD. Garlic: a review of its medicinal effects and indicated active compounds. In: Phytomedicines of Europe. Chemistry and Biological Activity. Series 691 (Edited by: Lawson LD \& Bauer R) American Chemical Society. Washington, DC; 1998. p. 176-209.

10. Woodward PW. Garlic and friends: the history, growth and use of edible alliums. Melbourne: Hyland House; 1996. p. 2-22.

11. USDA. Department of Agriculture, Agricultural Research Service. [Internet] 2013. National Nutrient Database for Standard Reference, Release 25. Nutrient Data Laboratory. [acesso em: 2015 set 02]. Disponível em: http://www.ars. usda.gov/ba/bhnrc/ndl. 
12. Ali M, Thomson M, Afzal M. Garlic and onions: Their effect on eicosanoid metabolism and its clinical relevance. Prostglandins Leukot Essent Fatty Acids. 2000;62(2):55-73.

13. Bonzin B, Minica-dukic N, Samojlik I, Goran A, Igic R. Phenolics as antioxidants in garlic (Alium sativum L., Alliaceae). Food Chem. 2008;111:925-9.

14. Thomson M, Mustafa T, Ali M.Tromboxane-B2 levels in serum of rabbits receiving a single intravenous dose of aqueous extract of garlic and onion. Prostglandins Leukot Essent Fatty Acids. 2000;63:217-21.

15. Anwar MM, Meki AR. Oxidative stress in streptozotocin-induced diabetic rats: effects of garlic oil and meltonin. Comp BiochemPhysiol A MolIntegr Physiol 2003;135:539-547.

16. Bakri IM, Douglas CW. Inibitory effect of garlic extract on oral bacteria. Arch Oral Biol. 2005;50:645-51.

17. Ali M, Al-Qattan KK, Al Enezi F, Khanafer RM, Mustafa T. Effect of allicin from garlic powder on serum lipids and blood pressure in rats fed with a high cholesterol diet. Prostglandins Leukot Essent Fatty Acids. 2000;62(4):253-9.

18. Augusti K.T. Terapeutic values of onion (Alluimcepa L.) and garlic (Allium sativum L.). Indian J Exp Biol. 1996;34(7):634-40.

19. Banerjee SK, Maulik SK. Effect of garlic on cardiovascular disorders: a review. Nutr J. 2002;1(4):1-14.

20. Gómez LJG, Sánchez-Muniz FJ. Revisión: Efectos cardiovasculares Del ajo (Allium sativum). Arch Latinoam Nutr. 2000;50(3):219-29.

21. Jain AK, Vargas R, Gotzkowsky S, Mcmahon FG. Can garlic reduce levels of serum lipids? A controlled clinical study. Am J Med. 1993;94(6):632-5.

22. Steiner M, Khan AH, Holbert D, Lin RI. A double-blind crossover study in moderately hypercholesterolemic men that compared the effect of aged garlic extract and placebo administration on blood lipids. Am J Clin Nutr. 1996;64:866-70.

23. Adler AJ, Holub BJ. Effect of garlic and fish-oil supplementation on serum lipid and lipoprotein concentrations in hypercholesterolemic. Am J Clin Nutr. 1997;65:445-50.

24. Garden CD, Chatterjee LM, Carlson JJ. The effect of a garlic preparation on plasma lipid levels in moderately hypercholesterolemic adults. Atherosclerosis. 2001;154(1):213-20.
25. Kannar D, Wattanapenpaiboon N, Savige GS, Wahlqvist ML. Hypocholesterolemic effect of an enteric-coated garlic supplement. J Am Coll Nutr. 2001;20(3):225-31.

26. Peleg A, Hershcovici T, Lipa R, Anbar R, Redler M, BeigelY. Effect of garic on lipid profile and psychopathologic parameters in people with mild to moderate hypercholesterolemia. IMAJ 2003;5:637-40.

27. Satitvipawee P, Rawdaree P, Indrabhakti S, Ratanasuwan T, Getn-gern P, Viwatwongkasem C. No effect of garlic extract supplement on serum lipid levels in hypercholesterolemic subjects. J Med Assoc Thai. 2003;86(8):750-7.

28. Tanamai J, Veeramanomai S, Indrakosas N. The efficacy of cholesterol-lowering action and side effects of garlic enteric coated tablets in man. J Med Assoc Thai. 2004;87(10):1156-61.

29. Sumioka I, Hayama M, Shimokawa Y, Shiraishi S, Tokunaga A. Lipid-lowering effect of monascus garlic fermented extract (MGFE) in hyperlipidemic subjects. Hiroshima J Med Sci. 2006;55(2):59-64.

30. Macan H, Uykimpang R, Alconcel M, Takasu J, Razon $\mathrm{R}$, Amagase H, et al. Significance of garlic and its constituents in cancer cardiovascular disease: aged garlic extract may be safe for patients on warfarin therapy. J Nutr. 2006;136:793-5.

31. Gardner CD, Lawson LD, Block E, Chatterjee LM, Kiazand A, Balise RR, et al. Effect of raw garlic vs commercial garlic supplements on plasma lipid concentrations in adults with moderate hypercholesterolemia: a randomized clinical trial. Arch Intern Med. 2007;167(4):346-53.

32. Kojuri J, Vosoughi AR, Akrami M. Effects of anethum graveolens and garlic on lipid profile in hyperlipidemic patients. Lipids Health Dis. 2007;1:6-5.

33. Sobenin IA, Adrianova IV, Demidova ON, Gorchakova TV, Orekhov AN. Lipid-lowering effects of time-released garlic powder tablets in double-blinded placebo-controlled randomized study. J Atheroscler Thromb. 2008; 15:334-8.

34. Jung ES, Park SH, Choi EK, Ryu BH, Park BH, Kim DS, et al. Reduction of blood lipid parameters by a 12 -wk supplementation of aged black garlic: a randomized controlled trial. Nutrition. 2014;30(9):1034-9.

\section{Como citar este artigo:}

Silva PL, Silva EM, Carmo MGT, Cardoso FS. Fitoterapia, allium sativum e hipercolesterolemia: uma revisão. Rev. Aten. Saúde. 2016;14(49):78-83. 